Strahlenther Onkol 2013 · 189:261-261

DOI 10.1007/s00066-012-0301-2

Online publiziert: 16. Januar 2013

(c) Springer-Verlag Berlin Heidelberg 2013

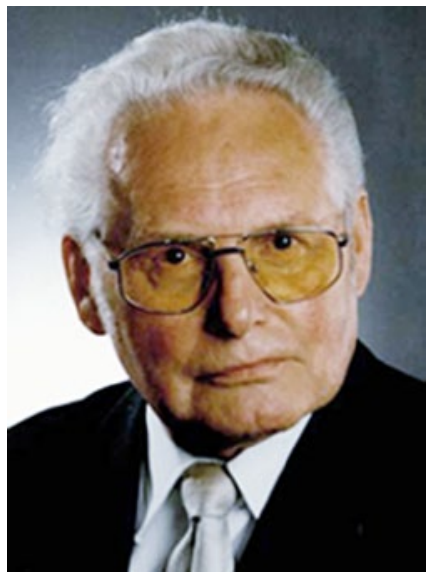

Professor Manfred Strietzel (Mit freundlicher Genehmigung durch Prof. Dr. M. Strietzel)

Professor Manfred Strietzel wurde in Kleinschönau/Niederschlesien geboren, legte das Abitur in Zittau ab und studierte Humanmedizin in Leipzig. Nach einer internistischen Ausbildung im BergmannsKrankenhaus Klettwitz begann er 1956 seine Ausbildung in der Radiologie und war ab 1959 an der Medizinischen Akademie Dresden in diesem Fache tätig. Nach seinem zweiten Facharztabschluss wechselte er dauerhaft in die Strahlentherapie und baute eine der größten ostdeutschen radioonkologischen Abteilungen in Dresden auf, die er als Abteilungsleiter der radiologischen Klinik bis 1978 leitete. In diesem Jahr wurde er anfangs zum Hochschuldozenten, später zum Professor für Strahlentherapie mit eigener großer Klinik an die medizinische Fakultät der Universität Rostock berufen. Dort konnte er

Th. Herrmann · J. Schorcht

Dresden

\title{
Professor Manfred Strietzel zum 85. Geburtstag
}

\author{
Professor Manfred Strietzel on the \\ occasion of his 85th birthday
}

in den Jahren ab 1989 an der Umgestaltung und Demokratisierung dieser Hochschule in verantwortlicher Position mitwirken, bevor er 1996 emeritiert wurde.

Professor Strietzels wesentliches Verdienst besteht darin, dass er die Strahlentherapie stets als interdisziplinäres Fach im Umfeld anderer onkologischer Disziplinen gesehen hat und entsprechende Strukturen zur gemeinsamen onkologischen Arbeit schuf. Schon 1968 gründete er an der Dresdner Klinik mit der MundKiefer-Gesichtschirurgie eine regelmäBige interdisziplinäre Sprechstunde, der später weitere Konzile mit anderen Kliniken folgten. In einem in der DDR erschienenen Studentenlehrbuch, für dessen Strahlentherapieteil Prof. Strietzel verantwortlich war, wurde - durch die Dresdner Erfahrungen begründet - auf die Darstellung interdisziplinärer Krebsbehandlung großer Wert gelegt und dies auch durch entsprechende Autorenschaft belegt. Diesen interdisziplinären Ansatz übertrug er auch auf seine Arbeit in Rostock und konnte ihn als Leiter des Tumorzentrums Rostock von 1992 bis 1998 weiterentwickeln.

Professor Strietzel übernahm 1984 die Chefredaktion der Zeitschrift Radiobiologia Radiotherapia, die er bis zu deren Aufgabe im Jahre 1990 leitete. Nachdem er unter den schwierigen Bedingungen der damaligen Zeit in Rostock eine moderne Klinik aufgebaut hatte, beschäftigte er sich in seinen letzten Berufsjahren intensiv mit Brachytherapie, insbesondere mit den neuen Möglichkeiten der Afterloading-Therapie. Er konnte an der Rosto- cker Einrichtung eine leistungsfähige Abteilung für diese Therapieform schaffen.

Professor Strietzel hat die ostdeutsche Strahlentherapie unzweifelhaft geprägt. Insbesondere in seiner Tätigkeit als Chefredakteur der ostdeutschen Fachzeitschrift kam ihm seine absolute Exaktheit und größte Genauigkeit in wissenschaftlichen und publizistischen Fragen zugute. Diese besondere Korrektheit, aber auch seine mitfühlende Zuwendung zum Tumorpatienten sowie seine konsequente menschliche und undoktrinäre Haltung auch gegenüber Kollegen und Mitarbeitern sind für viele seiner Schüler Vorbild für ihr eigenes Wirken in der Radioonkologie geworden.

Anfang Mai 2013 wird Manfred Strietzel sein 85 . Lebensjahr vollenden. Seine Schüler danken ihm für seinen immerwährenden ausgewogenen Rat, den sie auch einfordern konnten, nachdem sie selbst schon in verantwortlichen Positionen waren, und wünschen ihm Gesundheit, verbunden mit der Zusage, dass er in Dresden weiterhin stets ein gern gesehener Gast ist und dies nicht nur, wenn er als Ehrenmitglied der Sächsischen Radiologischen Gesellschaft deren Jahrestagungen besucht.

Thomas Herrmann und

Johannes Schorcht, Dresden

\section{Korrespondenzadresse}

Prof. Dr. Th. Herrmann

Elbeweg 8, 01326 Dresden

Th.Herrmann@macbay.de 